\title{
Metal Chalcogenide Supertetrahedral Clusters: Synthetic Control over Assembly, Dispersibility and Their Functional Applications
}

\author{
JIAXU ZHANG, ${ }^{\dagger}$ XIANHUI BU,${ }^{\ddagger}$ PINGYUN FENG ${ }^{\S}$ AND TAO WU ${ }^{*}, \uparrow$ \\ College of Chemistry, Chemical Engineering and Material Sciences, Soochow University, \\ Suzhou, Jiangsu 215123, China \\ * Department of Chemistry and Biochemistry, California State University, Long Beach, \\ California 90840, United States \\ $\S$ Department of Chemistry, University of California, Riverside, California 92521, United States
}

* corresponding author email: wutao@suda.edu.cn 
Table S1. The synthesis, yield, stability and solubility of discrete MCSCs

\begin{tabular}{|c|c|c|c|c|c|}
\hline $\begin{array}{l}\text { Discrete } \\
\text { MCSCs }\end{array}$ & Synthesis & Yield & Stability & Solubility & Ref. \\
\hline \multirow{7}{*}{$\begin{array}{c}\text { T4-MnGaSnS } \\
\text { (OCF-40) }\end{array}$} & $\mathrm{Mn}(\mathrm{Ac})_{2} \cdot 4 \mathrm{H}_{2} \mathrm{O}(34 \mathrm{mg}, 0.139 \mathrm{mmol})$ & \multirow{7}{*}{$105.2 \mathrm{mg}$} & \multirow{7}{*}{ Air-stable } & \multirow{7}{*}{ PR } & \multirow{7}{*}{$1-3$} \\
\hline & $\mathrm{Ga}\left(\mathrm{NO}_{3}\right)_{3} \cdot x \mathrm{H}_{2} \mathrm{O}(272.0 \mathrm{mg}, 1.062 \mathrm{mmol})$ & & & & \\
\hline & $\mathrm{SnCl}_{2}(30.0 \mathrm{mg}, 0.158 \mathrm{mmol})$ & & & & \\
\hline & $\mathrm{S}(76.0 \mathrm{mg}, 2.371 \mathrm{mmol})$ & & & & \\
\hline & $\mathrm{H}_{2} \mathrm{O}(2.481 \mathrm{~g}, 137.8 \mathrm{mmol})$ & & & & \\
\hline & PR (2.023 g, $23.75 \mathrm{mmol})$ & & & & \\
\hline & $200{ }^{\circ} \mathrm{C}, 10$ days & & & & \\
\hline \multirow{8}{*}{$\begin{array}{c}\text { Mn doped } \\
\text { T4-ZnGaSnS } \\
(\mathrm{Zn}: \mathrm{Mn}=2.89: \\
1.11) \\
(\text { OCF-40) }\end{array}$} & $\mathrm{Mn}(\mathrm{Ac})_{2} \cdot 4 \mathrm{H}_{2} \mathrm{O}(28 \mathrm{mg})$ & \multirow{8}{*}{$\mathbf{N} / \mathbf{A}$} & \multirow{8}{*}{ Air-stable } & \multirow{8}{*}{ PR } & \multirow{8}{*}{$1-3$} \\
\hline & $\mathrm{Zn}\left(\mathrm{NO}_{3}\right)_{2} \cdot 6 \mathrm{H}_{2} \mathrm{O}(76.9 \mathrm{mg}, 0.259 \mathrm{mmol})$ & & & & \\
\hline & $\mathrm{Ga}\left(\mathrm{NO}_{3}\right)_{3} \cdot x \mathrm{H}_{2} \mathrm{O}(253.0 \mathrm{mg}, 0.988 \mathrm{mmol})$ & & & & \\
\hline & $\mathrm{SnCl}_{2}(35.0 \mathrm{mg}, 0.184 \mathrm{mmol})$ & & & & \\
\hline & $\mathrm{S}$ (72 mg, $2.245 \mathrm{mmol})$ & & & & \\
\hline & $\mathrm{H}_{2} \mathrm{O}(2.481 \mathrm{~g}, 137.8 \mathrm{mmol})$ & & & & \\
\hline & PR (2.023 g, $23.75 \mathrm{mmol})$ & & & & \\
\hline & $200{ }^{\circ} \mathrm{C}, 10$ days & & & & \\
\hline \multirow{4}{*}{$\begin{array}{c}\text { T4-CdInSCI* } \\
\text { (T4-1) }\end{array}$} & $1 / 10$ of the precursor solution- 1 (wt $\%$ ) & \multirow{4}{*}{$\begin{array}{c}87.8 \mathrm{mg} \\
31 \% \text { (In) }\end{array}$} & \multirow{4}{*}{ Air-stable } & \multirow{4}{*}{$\mathbf{N} / \mathbf{A}$} & \multirow{4}{*}{4} \\
\hline & $\mathrm{CdCl}_{2}(91.5 \mathrm{mg}, 0.5 \mathrm{mmol})$ & & & & \\
\hline & DBN (0.500 g) & & & & \\
\hline & $140{ }^{\circ} \mathrm{C}, 5$ days & & & & \\
\hline \multirow{4}{*}{$\begin{array}{c}\text { T4-CdInSSeCl* } \\
\text { (T4-2) }\end{array}$} & $1 / 10$ of the precursor solution- 2 (wt $\%)$ & \multirow{4}{*}{$\begin{array}{c}144.6 \mathrm{mg} \\
43.5 \% \text { (In) }\end{array}$} & \multirow{4}{*}{ Air-stable } & \multirow{4}{*}{ DMSO } & \multirow{4}{*}{4} \\
\hline & $\mathrm{CdCl}_{2}(91.5 \mathrm{mg}, 0.5 \mathrm{mmol})$ & & & & \\
\hline & HTMP (2.000 g) & & & & \\
\hline & $140{ }^{\circ} \mathrm{C}, 5$ days. & & & & \\
\hline \multirow{4}{*}{$\begin{array}{c}\text { T4-CdInSeCl* } \\
\text { (T4-3) }\end{array}$} & $1 / 10$ of the precursor solution- $3(\mathrm{wt} \%)$ & \multirow{4}{*}{$\begin{array}{c}90 \mathrm{mg} \\
24 \% \text { (In) }\end{array}$} & \multirow{4}{*}{ Air-stable } & \multirow{4}{*}{ DMSO } & \multirow{4}{*}{4} \\
\hline & $\mathrm{CdCl}_{2}(91.5 \mathrm{mg}, 0.5 \mathrm{mmol})$ & & & & \\
\hline & 4,4'-bipyridyl (156 mg) & & & & \\
\hline & $140{ }^{\circ} \mathrm{C}, 5$ days & & & & \\
\hline \multirow{7}{*}{$\begin{array}{c}\text { T4-ZnInS } \\
\text { (ISC-16) }\end{array}$} & $\mathrm{Zn}(\mathrm{Ac})_{2} \cdot 2 \mathrm{H}_{2} \mathrm{O}(60 \mathrm{mg}, 0.273 \mathrm{mmol})$ & \multirow{7}{*}{$\sim 80 \mathrm{mg}$} & \multirow{7}{*}{ Air-stable } & \multirow{7}{*}{$\begin{array}{c}\mathrm{H}_{2} \mathrm{O}, \\
\text { DMF, } \\
\text { acetone, } \\
\mathrm{H}_{2} \mathrm{O} \text { and } \\
\text { ethanol }\end{array}$} & \\
\hline & In (44.0 mg, $0.383 \mathrm{mmol})$ & & & & \\
\hline & S (118 mg, 3.688 mmol) & & & & \\
\hline & $\mathrm{H}_{2} \mathrm{O}(1.0 \mathrm{~mL})$ & & & & 5 \\
\hline & DMP (3.0 mL) & & & & \\
\hline & DBU (1.0 mL) & & & & \\
\hline & $190{ }^{\circ} \mathrm{C}, 9$ days & & & & \\
\hline
\end{tabular}




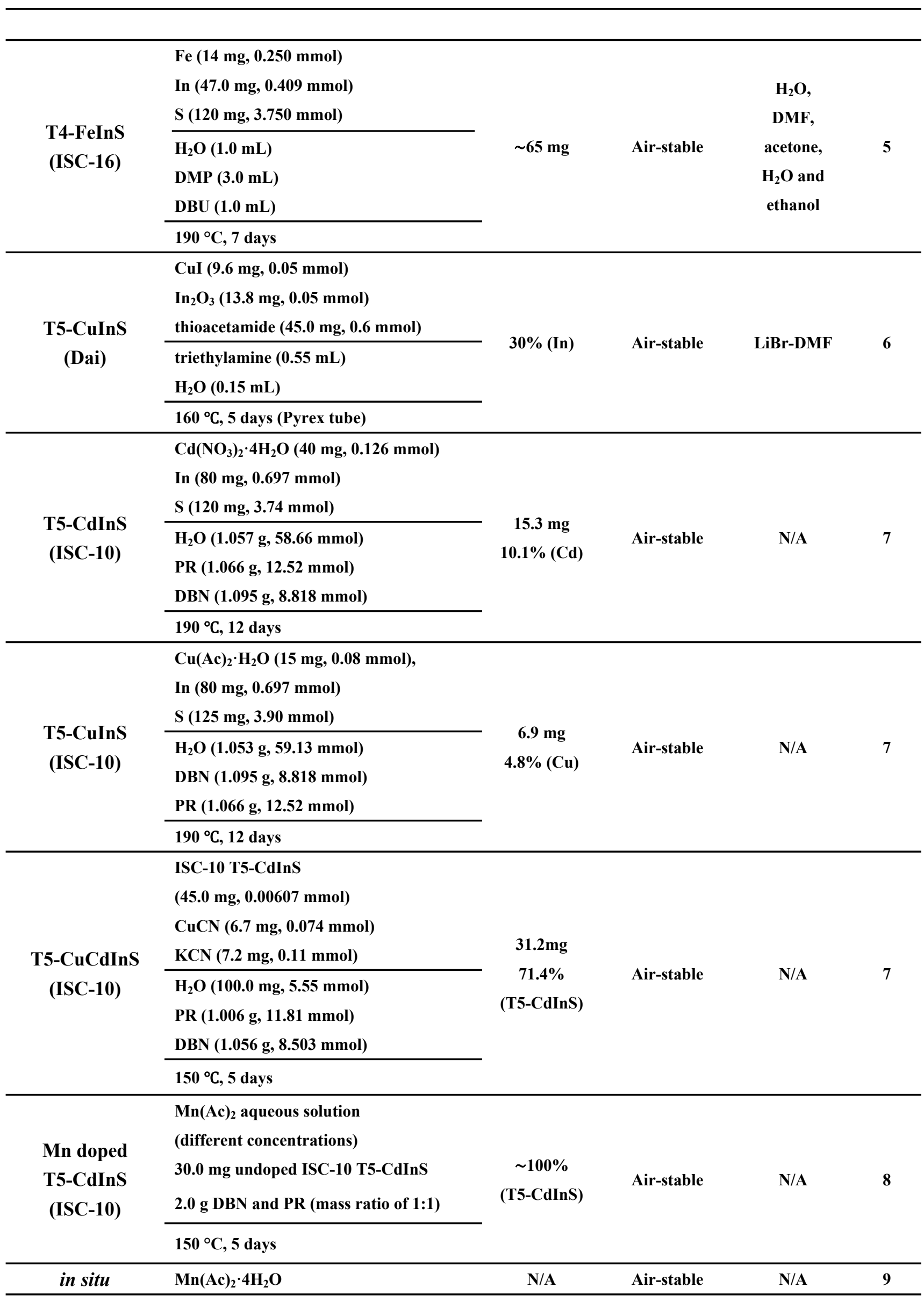




\begin{tabular}{|c|c|c|c|c|c|}
\hline \multirow{8}{*}{$\begin{array}{c}\text { Mn doped } \\
\text { T5-CdInS } \\
\text { (ISC-10) }\end{array}$} & $(1.4,2.8,5.5,11.0,16.5$, and $22.0 \mathrm{mg})$ & & & & \\
\hline & $\mathrm{Cd}\left(\mathrm{NO}_{3}\right)_{2} \cdot 4 \mathrm{H}_{2} \mathrm{O}(40 \mathrm{mg}, 0.126 \mathrm{mmol})$ & & & & \\
\hline & In (80 mg, $0.697 \mathrm{mmol})$ & & & & \\
\hline & $\mathrm{S}(120 \mathrm{mg}, 3.74 \mathrm{mmol})$ & & & & \\
\hline & $\mathrm{H}_{2} \mathrm{O}(1.0 \mathrm{~mL})$ & & & & \\
\hline & DBN (1.0 mL) & & & & \\
\hline & $\operatorname{PR}(1.2 \mathrm{~mL})$ & & & & \\
\hline & $200^{\circ} \mathrm{C}, 10$ days & & & & \\
\hline \multirow{6}{*}{ P2-CuInSnS } & $\mathrm{CuI}$ (40 mg, $0.21 \mathrm{mmol})$ & \multirow{6}{*}{$>80 \mathrm{mg}$} & \multirow{6}{*}{ Air-stable } & \multirow{6}{*}{$\begin{array}{c}\mathrm{H}_{2} \mathrm{O} / \text { acetone } \\
1: 1 \text { (v:v) }\end{array}$} & \multirow{6}{*}{10} \\
\hline & In (114 mg, 1 mmol) & & & & \\
\hline & Sn (60 mg, 0.5 mmol) & & & & \\
\hline & S (224 mg, 7 mmol) & & & & \\
\hline & $\mathrm{H}_{2} \mathrm{O} / \mathrm{AB} / \mathrm{DBN}(1: 1: 3$ in volume $)$ & & & & \\
\hline & $170^{\circ} \mathrm{C}, 7$ days & & & & \\
\hline \multirow{7}{*}{ P2-CuGaInSnS } & $\mathrm{CuI}$ (40 mg, $0.21 \mathrm{mmol})$ & \multirow{7}{*}{$>80 \mathrm{mg}$} & \multirow{7}{*}{ Air-stable } & \multirow{7}{*}{$\begin{array}{c}\mathrm{H}_{2} \mathrm{O} / \text { acetone } \\
1: 1 \text { (v:v) }\end{array}$} & \multirow{7}{*}{10} \\
\hline & $\mathrm{Ga}_{2} \mathrm{O}_{3}(57 \mathrm{mg}, 0.75 \mathrm{mmol})$ & & & & \\
\hline & $\mathrm{In}\left(\mathrm{NO}_{3}\right)_{3} \cdot 4.5 \mathrm{H}_{2} \mathrm{O}(136 \mathrm{mg}, 0.25 \mathrm{mmol})$ & & & & \\
\hline & Sn (60 mg, $0.5 \mathrm{mmol})$ & & & & \\
\hline & $\mathrm{S}(224 \mathrm{mg}, 7.0 \mathrm{mmol})$ & & & & \\
\hline & $\mathrm{H}_{2} \mathrm{O} / \mathrm{AB} / \mathrm{DBN}$ (1:1:3 in volume) & & & & \\
\hline & $180^{\circ} \mathrm{C}, 10$ days & & & & \\
\hline \multirow{6}{*}{ P2-CuGaSnS } & CuI (40 mg, $0.21 \mathrm{mmol})$ & \multirow{6}{*}{$>80 \mathrm{mg}$} & \multirow{6}{*}{ Air-stable } & \multirow{6}{*}{$\begin{array}{c}\mathrm{H}_{2} \mathrm{O} / \text { acetone } \\
1: 1 \text { (v:v) }\end{array}$} & \multirow{6}{*}{10} \\
\hline & $\mathrm{Ga}\left(\mathrm{NO}_{3}\right)_{3} \cdot x \mathrm{H}_{2} \mathrm{O}(273 \mathrm{mg}, 1.0 \mathrm{mmol})$ & & & & \\
\hline & Sn (60 mg, $0.5 \mathrm{mmol})$ & & & & \\
\hline & $\mathrm{S}(224 \mathrm{mg}, 7.0 \mathrm{mmol})$ & & & & \\
\hline & $\mathrm{H}_{2} \mathrm{O} / \mathrm{AB} / \mathrm{DBN}$ (1:1:3 in volume) & & & & \\
\hline & $180^{\circ} \mathrm{C}, 10$ days & & & & \\
\hline
\end{tabular}

PR: Piperidine, DBN: 1,5-diazabicyclo[4.3.0]non-5-ene, HTMP: 2,2,6,6-tetramethylpiperidine, DMP: 3,5-dimethylpiperidine, DBU: 1,8-Diazabicyclo[5.4.0]undec-7-ene, AB: (R)-(-)-2-amino-1-butanol.

* In-Q-ILs (Q = S, Se) Precursors. The preparation of In-QILs precursors was carried out via a microwave-assisted ionothermal process on a microwave reactor (Biotage Initiator EXP) with a maximum power of $100 \mathrm{~W}$. Generally, $1.140 \mathrm{~g}(10 \mathrm{mmol}) \mathrm{of}$ In, $0.702 \mathrm{~g}(22$ $\mathrm{mmol})$ of S (T4-1), $0.351 \mathrm{~g}(11 \mathrm{mmol})$ of S, and $0.892 \mathrm{~g}$ (11 mmol) of Se (T4-2) or $1.738 \mathrm{~g} \mathrm{(22} \mathrm{mmol)} \mathrm{of} \mathrm{Se} \mathrm{(T4-3)} \mathrm{were} \mathrm{mixed} \mathrm{with} 10$ $\mathrm{g}(55 \mathrm{mmol})$ of (BMMim)Cl in a $20 \mathrm{~mL}$ microwave quartz vessel. Then, the vessel was sealed and held at $100{ }^{\circ} \mathrm{C}$ in a water bath with magnetic stirring until forming a mash-like mixture. After that, the obtained mixture was heated in a microwave using a controlled program with magnetic stirring. Typically, the mixture was held at $150{ }^{\circ} \mathrm{C}$ for $5 \mathrm{~min}$ followed by $190{ }^{\circ} \mathrm{C}$ for 30 min and then cooled to room temperature. Finally, a dark-red gel was formed.

Precursor solution-1 for T4-CdInSCl (T4-1): Ten millimoles of the as-synthesized dark-red gel was diluted with $5 \mathrm{~mL}$ of 1 methylimidazole (1-Mim).

Precursor solution-2 for T4-CdInSSeCl (T4-2): Ten millimoles of the as-synthesized dark-red gel was diluted with $10 \mathrm{~mL}$ of $\mathrm{n}$ methylpyrrolidone.

Precursor solution-3 for T4-CdInSeCl (T4-3): Ten millimoles of the as-synthesized dark-red gel was diluted with $5 \mathrm{~mL}$ of DMF. 


\section{Reference}

(1) Wu, T.; Wang, L.; Bu, X.; Chau, V.; Feng, P. Largest Molecular Clusters in the Supertetrahedral Tn Series. J. Am. Chem. Soc. 2010, 132, 10823-10831.

(2) Zhang, Q.; Lin, J.; Yang, Y.-T.; Qin, Z.-Z.; Li, D.; Wang, S.; Liu, Y.; Zou, X.; Wu, Y.-B.; Wu, T. Exploring Mn²+-location-dependent red emission from (Mn/Zn)-Ga-Sn-S supertetrahedral nanoclusters with relatively precise dopant positions. J. Mater. Chem. C 2016, 4, 10435-10444.

(3) Liu, D.; Liu, Y.; Huang, P.; Zhu, C.; Kang, Z.; Shu, J.; Chen, M.; Zhu, X.; Guo, J.; Zhuge, L.; Bu, X.; Feng, P.; Wu, T. Highly Tunable Heterojunctions from Multimetallic Sulfide Nanoparticles and Silver Nanowires. Angew. Chem. Int. Ed. 2018, 57, 5374-5378.

(4) Hao, M.; Hu, Q.; Zhang, Y.; Luo, M.; Wang, Y.; Hu, B.; Li, J.; Huang, X. Soluble Supertetrahedral Chalcogenido T4 Clusters: High Stability and Enhanced Hydrogen Evolution Activities. Inorg. Chem. 2019, 58, 5126-5133.

(5) Xue, C.; Zhang, L.; Wang, X.; Hu, D.; Wang, X.-L.; Zhang, J.; Zhou, R.; Li, D.-S.; Su, H.; Wu, T. Enhanced Water Dispersibility of Discrete Chalcogenide Nanoclusters with Sodalite-Net Loose-Packing Pattern in Crystal Lattice. Inorg. Chem. 2020, DOI: 10.1021/acs.inorgchem. 0c00621.

(6) Li, Z.-Q.; Mo, C.-J.; Guo, Y.; Xu, N.-N.; Zhu, Q.-Y.; Dai, J. Discrete supertetrahedral CuInS nanoclusters and their application in fabrication of cluster-sensitized $\mathrm{TiO}_{2}$ photoelectrodes. J. Mater. Chem. A 2017, 5, 8519-8525.

(7) Wu, T.; Zhang, Q.; Hou, Y.; Wang, L.; Mao, C.; Zheng, S.-T.; Bu, X.; Feng, P. Monocopper Doping in Cd-In-S Supertetrahedral Nanocluster via Two-Step Strategy and Enhanced Photoelectric Response. J. Am. Chem. Soc. 2013, 135, 10250-10253

(8) Lin, J.; Zhang, Q.; Wang, L.; Liu, X.; Yan, W.; Wu, T.; Bu, X.; Feng, P. Atomically Precise Doping of Monomanganese Ion into Coreless Supertetrahedral Chalcogenide Nanocluster Inducing Unusual Red Shift in Mn ${ }^{2+}$ Emission. J. Am. Chem. Soc. 2014, 136, 47694779 .

(9) Lin, J.; Hu, D.-D.; Zhang, Q.; Li, D.-S.; Wu, T.; Bu, X.; Feng, P. Improving Photoluminescence Emission Efficiency of Nanocluster Based Materials by in Situ Doping Synthetic Strategy. J. Phys. Chem. C 2016, 120, 29390-29396.

(10) Zhang, J.; Qin, C.; Zhong, Y.; Wang, X.; Wang, W.; Hu, D.; Liu, X.; Xue, C.; Zhou, R.; Shen, L.; Song, Y.; Xu, D.; Lin, Z.; Guo, J.; Su, H.; Li, D.-S.; Wu, T. Atomically Precise Metal-Chalcogenide Semiconductor Molecular Nanoclusters with High Dispersibility: Designed Synthesis and Intracluster Photocarrier Dynamics. Nano Research, 2020 in press. 